\title{
Variablilidad de la presión arterial, hipertensión arterial nocturna y su asociación con tabaquismo
}

Carolina Acosta ORCID: 0000-0002-3973-0364 Postgrado de Medicina Interna. Clínica Médica 3. UdelaR.

Paola Sposito ORCID: 0000-0001-8715-8385 Internista. Diabetóloga. Profesor Adjunta Clínica Médica 3. UdelaR.

Verónica Torres Esteche

ORCID: 0000-0003-0847-0122

Internista. Neumóloga. Profesor Agregado Clínica Médica 3. UdelaR.

Florencia Sacchi

ORCID: 0000-0003-0204-7921 Asistente Clínica Médica 3. UdelaR.

Lucia Pomies

ORCID: 0000-0002-4407-0911

Residente de Medicina Interna.

Clínica Médica 3. UdelaR.

Maximiliano Pereda

ORCID: 0000-0002-6075-3632 Cardiólogo.

Sergio Viñas ORCID: 0000-0002-0657-2777 Cardiólogo. Jefe del Departamento de Cardiología MUCAM.

Enrique Soto

ORCID: 0000-0001-7831-4745 Cardiólogo. Jefe del Departamento de Cardiología CASMU

\author{
Blood pressure variability, nocturnal hypertension and its association with \\ smoking \\ Variabilidade da pressão arterial, hipertensão arterial noturna e sua \\ associação com o tabagismo
}

Resumen: Introducción. La hipertensión arterial y el tabaquismo son factores de riesgo independientes para el desarrollo de eventos cardiovasculares. El consumo de tabaco determina una elevación aguda de la presión arterial por acción del sistema simpático. Sin embargo los efectos a largo plazo son contradictorios. El aumento de la variabilidad de la presión arterial y la presencia de hipertensión arterial nocturna se asocia con eventos cardiovasculares adversos independientemente de los niveles de presión arterial. Objetivos. Evaluar la asociación entre tabaquismo e hipertensión arterial diurna, nocturna y variabilidad. Material y Métodos. Estudio analítico, observacional, transversal, multicéntrico, que incluye pacientes hipertensos mayores de 18 años con monitoreo ambulatorio de la presión arterial (MAPA). Resultados. Se incluyeron 391 pacientes, siendo 14.6\% fumadores. Se encontraron cifras de presión arterial diurna sistólica y diastólica más elevadas en pacientes tabaquistas ( $p=0.204, p=0.087$, respectivamente). Se observó una asociación significativa entre la hipertensión arterial nocturna y la presencia de diabetes mellitus e índice de masa corporal aumentado. No se encontró asociación entre el consumo de tabaco y los distintos patrones de variabilidad, así como tampoco con la hipertensión arterial nocturna. Conclusiones. El grupo de pacientes fumadores presentó una tendencia a cifras de presión arterial media diurna sistólica y diastólica más elevadas que los no fumadores, lo que podría sugerir que el tabaquismo incide en el control de cifras de presión arterial.

Palabras claves: Tabaquismo, hipertensión arterial, variabilidad de la presión arterial, hipertensión arterial nocturna.

Abstract: Introduction. Hypertension and smoking are independent risk factors for the development of cardiovascular events. Tobacco use causes an acute elevation of blood pressure due to the action of the sympathetic system. However, the long-term effects are contradictory. Increased variability in blood pressure and the presence of nocturnal arterial hypertension are associated with adverse cardiovascular events regardless of blood pressure levels. Objectives. To evaluate the association between smoking and daytime and nighttime arterial hypertension and variability. Material and Methods. Analytical, observational, cross-sectional, multicenter study, which includes hypertensive patients over 18 years of age with ambulatory blood pressure monitoring (ABPM). Results. 391 patients were included, being 14.6\% smokers. Higher levels of systolic and diastolic daytime blood pressure were found in smoking patients $(p=0.204, p=0.087$, respectively). A significant association was observed between nocturnal arterial hypertension and the presence of diabetes mellitus and increased body mass index. No association was found between tobacco consumption and the different patterns of variability, as well as with nocturnal arterial hypertension. Conclusions. The group of smoking patients showed a trend towards higher mean daytime systolic and diastolic blood pressure figures than non-smokers, which could suggest that smoking affects the control of blood pressure numbers.

Key words: Smoking, arterial hypertension, blood pressure variability, nocturnal hypertension. 
Resumo: Introdução. Hipertensão e tabagismo são fatores de risco independentes para o desenvolvimento de eventos cardiovasculares. O uso do tabaco provoca elevação aguda da pressão arterial devido à ação do sistema simpático. No entanto, os efeitos de longo prazo são contraditórios. O aumento da variabilidade da pressão arterial e a presença de hipertensão arterial noturna estão associados a eventos cardiovasculares adversos, independentemente dos níveis de pressão arterial. Objetivos. Avaliar a associação entre tabagismo e hipertensão arterial diurna e noturna e variabilidade. Materiai e Métodos. Estudo analítico, observacional, transversal, multicêntrico, que inclui hipertensos maiores de 18 anos com monitorização ambulatorial da pressão arterial (MAPA). Resultados. Foram incluídos 391 pacientes, sendo $14,6 \%$ tabagistas. Níveis mais elevados de pressão arterial diurna sistólica e diastólica foram encontrados em pacientes fumantes ( $p=0,204, p=0,087$, respectivamente). Foi observada associação significativa entre hipertensão arterial noturna e presença de diabetes mellitus e aumento do índice de massa corporal. Não foi encontrada associação entre o consumo de tabaco e os diferentes padrões de variabilidade, bem como com a hipertensão arterial noturna. Conclusões. O grupo de pacientes fumantes apresentou tendência a valores médios de pressão arterial sistólica e diastólica mais elevados do que os não fumantes, o que pode sugerir que o tabagismo afeta o controle dos valores da pressão arterial.

Palavras-chave: Tabagismo, hipertensão arterial, variabilidade da pressão arterial, hipertensão arterial noturna 


\section{Introducción}

Tabaquismo e hipertensión arterial (HTA) constituyen dos de los principales factores de riesgo cardiovascular y el estudio Framingham demostró que la asociación de ambos determina un aumento del riesgo 4 veces mayor ${ }^{(1)}$.

\section{A) Tabaquismo}

Es un factor de riesgo cardiovascular prevalente en la población, según la Organización Mundial de la Salud (OMS) causa ocho millones de muerte en el mundo cada año (2).

Las tasas de tabaquismo actuales se encuentran en descenso en Uruguay de acuerdo a última encuesta GATS, siendo del $21,6 \%{ }^{(3)}$. A pesar de ello, existe un incremento del uso de dispositivos electrónicos de liberación de nicotina, como el cigarrillo electrónico y los HTPS (heath tobacco product system) así como también un aumento del consumo dual de cannabis y tabaco. Como consecuencia el consumo de tabaco se mantiene como uno de los problemas más importantes de la salud pública ${ }^{(4)}$.

El humo del cigarrillo contiene aproximadamente 7000 sustancias químicas, muchas de las cuales aumentan el estrés oxidativo, la disfunción endotelial y el remodelado cardíaco, colaborando en el desarrollo de la aterosclerosis y aumentando el riesgo cardiovascular ${ }^{(5)}$.

\section{B) Hipertensión arterial}

Es una patología muy prevalente, se calcula que la padecen entre un $30-45 \%$ de los adultos (6). Existe una asociación independiente entre la edad y el desarrollo de HTA ${ }^{(7)}$. La HTA representa un factor de riesgo independiente para el desarrollo de eventos cardiovasculares y de mortalidad, y suele acompañarse de otros factores de riesgo asociados que aumentan la morbi-mortalidad de los pacientes ${ }^{(8)}$.

\section{C) Otros factores de riesgo}

Entre los factores de riesgo asociados a la HTA se destacarán el sobrepeso-obesidad, el síndrome de apnea-hipoapnea del sueño y la diabetes mellitus.

Sobrepeso-obesidad: El aumento excesivo de peso se asocia con el desarrollo de HTA. Se observó en algunos estudios, una relación directa entre el índice de masa corporal y la hipertensión arterial.

Síndrome de apnea-hipoapnea del sueño (SAHOS): Estudios observacionales han demostrado que el SAHOS aumenta la incidencia de hipertensión ${ }^{\left({ }^{9}\right)}$. Los episodios de apnea hipopnea que presentan los individuos durante la noche, generan un aumento de la presión arterial nocturna y se asocian a la presencia de HTA diurna, que se da en aproximadamente un $60 \%$ de los pacientes con SAHOS ${ }^{(10)}$. Es una comorbilidad altamente prevalente $(>80 \%)$ en los pacientes con HTA resistente. ${ }^{(9)}$.

Diabetes Mellitus (DM): La HTA se asocia con frecuencia en el paciente con DM teniendo una prevalencia de un $80 \%$. Se ha observado mayores dificultades en el tratamiento de la HTA en los pacientes que presentan diabetes, requiriendo tratamiento combinado en la mayoría de los $\operatorname{casos}^{(9)}$.

\section{D) Variabilidad de la presión arterial}

Las cifras de presión arterial presentan variabilidad durante las 24 horas del día, observándose condiciones normales un descenso en la noche, durante el sueño. Esta variabilidad está afectada por diferentes factores biológicos, comportamentales y ambientales.

Los diferentes patrones de variabilidad se definen por el porcentaje de descenso de los valores medios de la presión arterial (PA) de la noche (período de sueño) en relación al día (actividad al despertar) ${ }^{(11)}$

En la tabla 1 se presentan los patrones de variabilidad de la PA.

\begin{tabular}{|l|l|}
\hline Patrón de variabilidad & Cambio en la media de presión arterial del período de sueño en relación al día. \\
\hline Patrón non-dipper & Descenso $<10 \%$ \\
\hline
\end{tabular}


Tabla 1: Patrones de variabilidad de la presión arterial. Modificada de Sauza-Sosa JC ${ }^{(12)}$

\begin{tabular}{|l|l|}
\hline Patrón dipper & Descenso $10-20 \%$ \\
\hline Patrón dipper extremo & Descenso $>20 \%$ \\
\hline Patrón riser & Aumento de la presión arterial \\
\hline
\end{tabular}

La incorporación del Monitoreo Ambulatorio de la PA (MAPA), ha permitido establecer patrones de PA en función de su variabilidad circadiana, estableciendo perfiles de riesgo. La ausencia de caída nocturna fisiológica de la PA (patrón non dipper) o incluso su ascenso (patrón Riser) se han asociado a mayor frecuencia de eventos cardiovasculares adversos y daño orgánico en forma precoz ${ }^{(13)}$.

En los últimos años, se ha estudiado de manera extensa el papel de la variabilidad de la PA como factor de riesgo de enfermedad cardiovascular. Varios estudios observaron que el aumento de variabilidad de la PA, se asocia con eventos cardiovasculares adversos, independientemente del nivel de PA ${ }^{(12)}$. En estos estudios, los individuos se evaluaron mediante una medición aislada de la presión arterial de acuerdo con las recomendaciones de las directrices nacionales e internacionales ${ }^{(14)}$.

Se ha postulado a la HTA nocturna como mejor predictor de riesgo cardiovascular, independientemente del patrón circadiano ${ }^{(11)}$. Se define HTA nocturna a los valores $\geq 120 / 70$ $\mathrm{mmHg}(6)$.

\section{E) Relación entre tabaco y control de la HTA}

El consumo de tabaco causa una elevación aguda de la PA y de la frecuencia cardiaca por acción del sistema simpático, sin embargo, los efectos a largo plazo no están bien establecidos.

En este sentido, los resultados de los estudios son contradictorios. En algunos estudios se observó un aumento crónico de las cifras de PA en los fumadores ${ }^{(15)}$ mientras que en otros, se observaron cifras más bajas de PA en los pacientes tabaquistas comparados a los pacientes que no consumen tabaco ${ }^{(16)}$.

Hay evidencia de que el consumo de tabaco causa un aumento temporal en los niveles de PA tanto para individuos hipertensos como normotensos. Los estudios epidemiológicos que evalúan los niveles de PA utilizando mediciones casuales de la misma en el consultorio han demostrado que los fumadores presentan lecturas de PA menores o iguales a las de los sujetos no fumadores ${ }^{(17,18)}$

Con la hipótesis que el tabaquismo podría asociarse a un patrón de variabilidad de PA que predice mayor riesgo cardiovascular y lo a HTA nocturna, es que se realiza el presente trabajo con el objetivo principal de evaluar la asociación entre tabaquismo e HTA diurna y nocturna, así como evaluar la asociación entre tabaquismo y la variabilidad de la PA durante un período de 24 horas.

\section{Material y métodos}

Estudio analítico, observacional, transversal, multicéntrico (tres centros asistenciales).

Criterios de inclusión: mayores de 18 años hipertensos que se hayan realizado MAPA en un periodo de 3 meses.

Criterios de exclusión: embarazadas y pacientes que no pudieron completar el MAPA por motivos técnicos (calidad inferior, duración de menos de 21 horas o exclusiones automáticas superiores al $20 \%)$.

Se consideraron las siguientes variables: edad, sexo, consumo de tabaco, Índice paquete año de tabaco (IPA), diagnóstico previo de DM, SAHOS, índice de masa corporal (IMC), grupo de fármacos antihipertensivos, monoterapia, terapia combinada y cronoterapia.

Se evaluaron patrones de variabilidad, PAS y PAD diurna, hipertensión arterial nocturna.

Los pacientes fueron clasificados como fumadores o no fumadores de acuerdo con la información proporcionada por el paciente cuando se instaló el dispositivo.

El paciente que fuma un cigarrillo o más por día fue clasificado como fumador. 
Se definió la intensidad de consumo de tabaco por medio del IPA, el mismo se calculó como número de cajas de cigarrillos fumados al día por año de fumador.

Se analizaron los siguientes parámetros en el MAPA: lecturas medias de presión arterial sistólica y diastólica de 24 horas, diurnas y nocturnas; cargas de presión arterial sistólica y diastólica durante el día, la noche y las 24 horas. Se utilizó el monitor ABP oscilométrico de 24 horas Dyna-MAPA que ha sido validado por la Asociación Americana para el Avance de la Instrumentación Médica (AAMI) y la Sociedad Británica de Hipertensión (BHS). El software de programación y generación de informes también fue DynaMAPA.

El programa produce automáticamente el informe estadístico. El monitoreo se realizó en un día laborable. El programa del dispositivo registró mediciones cada 15 minutos durante el día y cada 20 minutos durante la noche. Los pacientes llevaron un diario de sus actividades y registro de los medicamentos que recibían.

Si tenían algún síntoma, debían registrarlos con la hora de inicio y conclusión. El día se consideró como el período entre las 7 a.m. y 11p.m. y noche las horas de sueño del paciente.

Se consideró cronoterapia la indicación de fármacos antihipertensivos previos a las horas de sueño.

Para el análisis estadístico se utilizó test chi cuadrado para el estudio de asociación entre variables cualitativas y test $T$ de Student para muestras independientes para el estudio de diferencias de variables continuas entre grupos. Previo se estudió la normalidad de las variables continuas con test de Kolmogorov - Smirnov. Se fijó un nivel de significación en alfa 0,05. El software utilizado correspondió a STATA v.12.0.

El estudio fue aprobado por los respectivos Comités de Ética de las tres instituciones participantes en las siguientes fechas: 25/01/2019, 24/04/2019, 15/04/2019 (Exp No 2019/0307)

\section{Resultados}

Se incluyeron en el estudio un total de 391 pacientes hipertensos.

En la tabla 2 se presentan las características epidemiológicas, presencia de factores de riesgo, patrones de HTA y tratamiento farmacológico recibido.

\begin{tabular}{|c|c|c|c|}
\hline Característica & F. Absoluta & F. Relativa \% & Media +/- EE \\
\hline $\begin{array}{ll}\text { Centro de } & \\
\text { Procedencia: } & 1 \\
& 2 \\
& 3\end{array}$ & $\begin{array}{r}213 \\
57 \\
121\end{array}$ & $\begin{array}{l}54,5 \\
14,6 \\
30,9\end{array}$ & \\
\hline $\begin{array}{l}\text { Sexo: Femenino } \\
\text { Masculino }\end{array}$ & $\begin{array}{l}256 \\
135\end{array}$ & $\begin{array}{l}65,5 \\
34,5\end{array}$ & \\
\hline Edad & & & $53,5+/-0,8$ \\
\hline Fuma actualmente & 57 & 14,6 & \\
\hline Edad de inicio al tabaquismo & \multirow{2}{*}{\multicolumn{2}{|c|}{$23,3+/-3,3$}} & $18,7+/-1,0$ \\
\hline IPA (índice de paquete año) & & & \\
\hline
\end{tabular}


Tabla 2: Características de los pacientes incluidos en el estudio. $\left({ }^{\star}\right)$ : Valores expresados en mediana (mínimo; máximo).

\begin{tabular}{|l|r|r|}
\hline DM & 62 & 15,9 \\
\hline SAHOS & 34 & 8,7 \\
\hline IMC: Normopeso & 81 & 20,8 \\
Sobrepeso & 150 & 38,4 \\
Obeso & 152 & 38,9 \\
\hline Patrones HTA: Dipper & 193 & 49,4 \\
Non dipper & 98 & 25,1 \\
Extreme dipper & 67 & 17,1 \\
Rising & 31 & 7,9 \\
\hline Cronoterapia & 119 & 30,4 \\
\hline HA nocturna & 225 & 57,5 \\
\hline Fármacos: Reciben fármacos & 241 & 61 \\
IECA & 97 & 24,8 \\
Betabloqueantes & 66 & 16,9 \\
ARA II & 122 & 31,2 \\
Calcioantagonistas & 75 & 19,2 \\
Diuréticos & 61 & 15,6 \\
Agonista alfa 2 & 8 & 2,0 \\
\hline
\end{tabular}

Se compararon hipertensos farmacológicamente tratados fumadores vs no fumadores.

Los parámetros MAPA se expresaron con valores de media estándar. La media de la PA sistólica durante el día, fue de $140,7 \pm 3,6 \mathrm{mmHg}$ en el grupo de fumadores vs $136,0 \pm 1,4$ $\mathrm{mmHg}$ en el grupo de no fumadores, no existiendo diferencias estadísticamente significativas entre ambos $(p=0,204)$. En el grupo de fumadores la media de la PA diastólica durante el día fue de $86,1 \pm 2,5 \mathrm{mmHg}$, y en el grupo de no fumadores fue de $82,3 \pm 0,8 \mathrm{mmHg}$, no existiendo diferencias estadísticamente significativas $(p=0,087)$. En la Gráfica 1 se presentan éstos resultados.

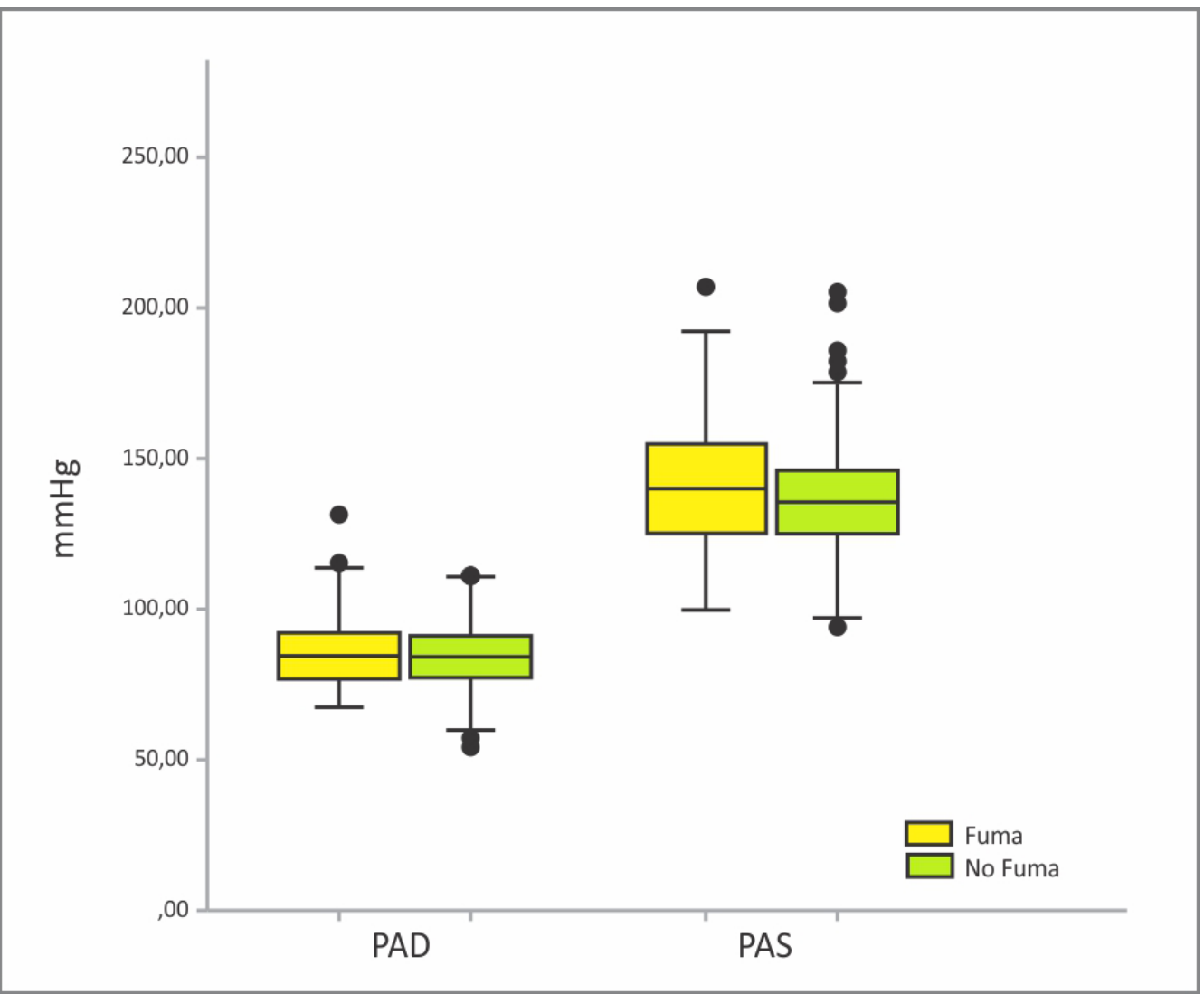


En la tabla 3 se presentan los resultados sobre los patrones de variabilidad de la PA hallados en el MAPA en ambos grupos de pacientes.

\begin{tabular}{|l|l|l|l|l|l|} 
& Dipper & Non dipper & Extreme dipper & Riser & Valor $p$ \\
\hline $\begin{array}{l}\text { Pacientes } \\
\text { fumadores }\end{array}$ & $50,9 \%(29)$ & $22,8 \%(13)$ & $17,6 \%(10)$ & $8,8 \%(5)$ & \\
\hline $\begin{array}{l}\text { Pacientes no } \\
\text { fumadores }\end{array}$ & $49,4 \%(164)$ & $25,6 \%(85)$ & $17,2(57)$ & $7,8 \%(26)$ & 0.973 \\
\hline
\end{tabular}

No se evidenció una asociación estadísticamente significativa entre HTA nocturna y tabaquismo. Sin embargo se evidenció una asociación estadísticamente significativa entre HTA nocturna con DM y/o un IMC aumentado ( $p=0,004$ y 0,001 respectivamente).

En la tabla 4 se presentan la relación de HTA nocturna con los factores de riesgo analizados y con el tratamiento farmacológico que reciben los pacientes

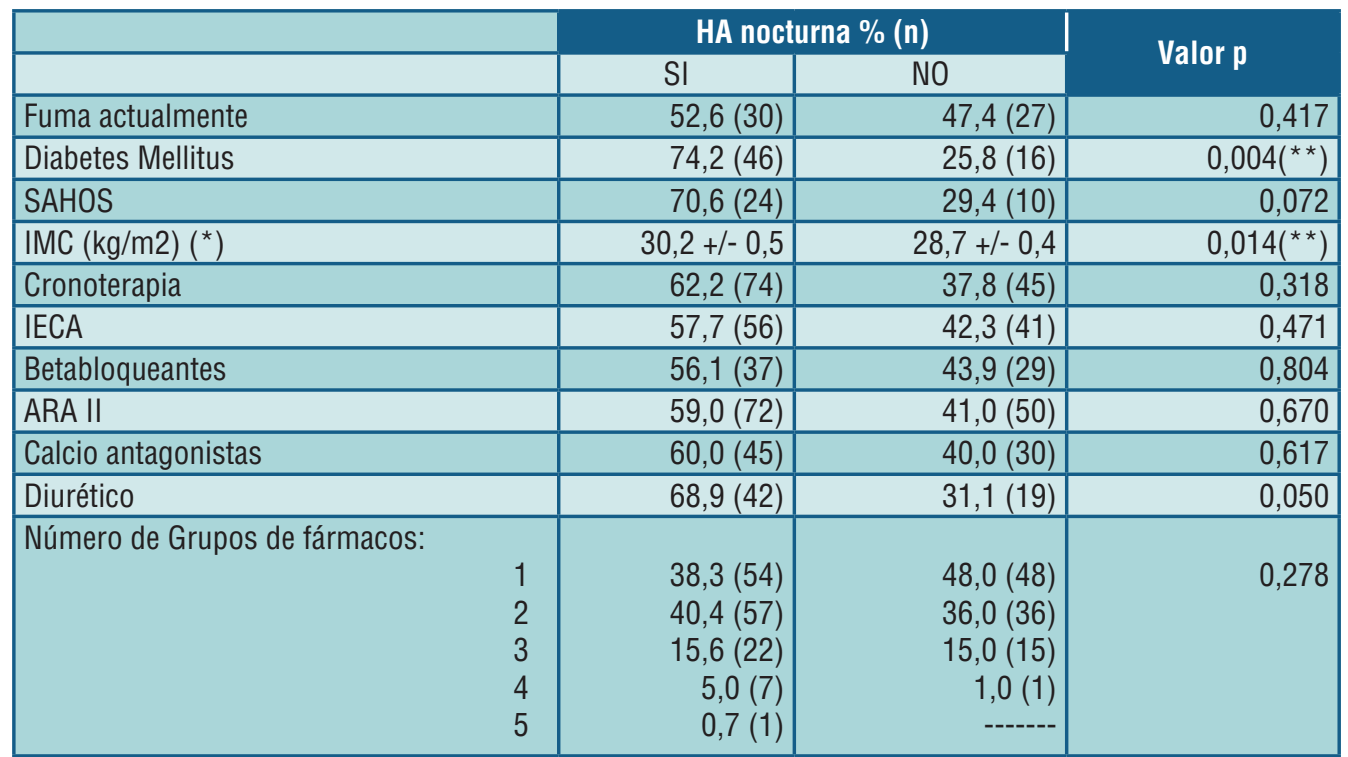

$\left.{ }^{*}\right)$ Expresado en media \pm error estándar ${ }^{* *}$ resultados estadísticamente significativos.

\section{Discusión}

\section{A) Género y edad}

Se encontró una prevalencia de sexo femenino (65,5\%), sobre el masculino $(34,5 \%)$, lo que podría estar vinculado a una mayor adherencia a estudios y tratamientos por parte del sexo femenino ${ }^{(19)}$. La HTA predomina en hombres a edades más tempranas, volviéndose más frecuente en mujeres a edades mayores, luego de la menopausia ${ }^{(20)}$.

\section{B) Tabaquismo}

La prevalencia de consumo de tabaco en este estudio fue del 14,6\%, siendo menor que los datos publicados en el estudio GATS, realizado en nuestro país en 2017 , donde el $21,6 \%$ de la población era fumadora (3). La menor prevalencia de tabaquismo, puede coincidir con la tendencia a la baja en el consumo de tabaco, luego de la implementación de políticas sanitarias para el control del mismo ${ }^{(21)}$.

\section{C) Tratamiento}

Se observó un número importante de pacientes tratados $(74,4 \%)$, siendo mayor que en otros estudios publicados a nivel nacional e internacional (9). Esto podría estar relacionado a que la población estudiada se encuentra en seguimiento con equipos de salud. En el estudio CESCAS I, realizado en el Cono Sur de América Latina, incluyendo a Uruguay, se observó que el $42 \%$ de 
los adultos presentaba HTA, encontrándose en conocimiento del diagnóstico solo el $63 \%$ de los mismos y bajo tratamiento el $48 \%$.

Se evidenció por otra parte que en el grupo de los pacientes bajo tratamiento $(61 \%$ del total de pacientes del estudio): $42 \%(102 / 241)$ recibían monoterapia y 57\% (139/241) terapia combinada. En el estudio CESCAS 1, estaban tratados con monoterapia $65 \%$, y con terapia combinada $35 \%{ }^{(22)}$.

\section{D) Cronoterapia}

A pesar de que ha sido demostrada la importancia de la cronoterapia en el tratamiento de la HTA y que existe una gran prevalencia de HTA nocturna entre los pacientes estudiados, solamente el $30,4 \%$ se encontraban bajo tratamiento farmacológico en la noche, probablemente debido a que el estudio fue solicitado en vistas a definir una conducta. El estudio Hygia Chronotherapy Trial, un estudio multicéntrico controlado, que incluyó a 19084 pacientes hipertensos, evidenció que la administración rutinaria de más de un fármaco antihipertensivo a la hora de dormir, en lugar de administrarlo al despertar, optimiza el control de la PA, con una disminución significativa de la PA nocturna y un incremento del descenso de PA durante el sueño, con disminución de eventos cardiovasculares ${ }^{(23)}$.

\section{E) Patrones de variabilidad}

El patrón de variabilidad más frecuente fue el patrón dipper $(49,4 \%)$, siguiendo en frecuencia el patrón non dipper $(25,1 \%)$, y con una prevalencia menor los patrones extreme dipper $(17,1 \%)$ y riser $(7,9 \%)$. La mayor prevalencia del patrón dipper, coincide con datos del Registro de Monitoreo de la Presión arterial Ambulatoria de la Sociedad Española de Hipertensión que evaluó un total de 43.000 pacientes hipertensos, siendo el patrón más frecuente dipper (50\% en pacientes sin tratamiento y $40 \%$ en pacientes con tratamiento) ${ }^{(24)}$.

\section{F) Asociación Tabaquismo y patrones de variabilidad}

En nuestro estudio, los patrones de variabilidad fueron similares en los pacientes tabaquistas, comparados a los pacientes no tabaquistas. Los resultados en otros estudios son contradictorios. Un estudio que incluyó 5235 pacientes, observó que el patrón non dipper era más prevalente en los pacientes fumadores ${ }^{(25)}$. Por el contrario, en otro estudio que incluyó a 1250 pacientes, el extreme dipper era más frecuente en los pacientes tabaquistas comparado a los no fumadores, siendo estas diferencias significativas ${ }^{(26)}$. La mayor prevalencia de patrón dipper y patrón extreme dipper en los pacientes fumadores, podría explicarse por una mayor diferencia de PA entre el día y la noche, dado fundamentalmente por un incremento de la presión diurna, sin afectarse las cifras de PA nocturna ${ }^{(27)}$.

\section{G) HTA nocturna}

La prevalencia de HTA nocturna fue de $57,7 \%$, similar a otros datos publicados ${ }^{(28)}$. En el Jackson Heart Study se observó una prevalencia de HTA nocturna del $57 \%$, mientras que en el Spanish ABPM Registry Study la prevalencia fue de $41 \%$ en los pacientes no tratados y de $50 \%$ en los pacientes tratados ${ }^{(29)}$.

\section{H) Asociación Tabaquismo e HTA nocturna}

No se observó asociación entre HTA nocturna y tabaquismo. La asociación entre estas dos variables, es controversial. Existen trabajos que hallaron una relación inversa entre HTA nocturna y tabaquismo, observando una menor PA nocturna en los pacientes fumadores $(30,31)$. Por el contrario, en el Spanish ABPM Registry Study $(n=99884)$, se encontró una asociación positiva entre HTA nocturna y tabaquismo, observándose además un mayor riesgo cardiovascular y un incremento de la mortalidad en los pacientes con HTA nocturna ${ }^{(29)}$. Finalmente, en otros estudios, no se encontró asociación entre estas dos variables. En un estudio caso control que comparó tabaquistas pesados (IPA mayor a 20) con sujetos no fumadores, no se observaron diferencias en los valores de PA nocturna entre los grupos ${ }^{(32)}$. En el estudio CARDIA y Jackson Heart Study, al analizar los datos de manera individual, se observó que la prevalencia de HTA nocturna fue mayor en los pacientes tabaquistas, sin embargo, al realizar un análisis estadístico multivariado de las cohortes, no se encontró asociación significativa entre estas variables ${ }^{(33)}$. 


\section{I) Asociación Tabaquismo e HTA diurna}

En cuanto a la relación entre HTA y tabaquismo, se observaron cifras de PA diurna más elevadas en pacientes tabaquistas, comparados a los no fumadores. Las diferencias fueron no significativas, lo que podría estar relacionado a la baja prevalencia de tabaquismo en la población estudiada y al tamaño muestral.

La relación entre tabaquismo e HTA es controversial. Algunos estudios hallaron una asociación positiva, con mayor incidencia de HTA tanto en hombres ${ }^{\left({ }^{(3)}\right.}$ como en mujeres tabaquistas ${ }^{(35)}$ con una media de PA sistólica y diastólica significativamente mayor en los pacientes fumadores, independientemente del uso de medicación antihipertensiva ${ }^{\left({ }^{36}\right)}$. Además se ha observado una PA diurna mayor en los días en que se consume tabaco, comparado a los días que no se consume ${ }^{(37)}$.

Estas cifras de PA elevada, podrían explicarse por la activación del sistema nervioso simpático y un incremento de las catecolaminas cuando aumentan los niveles de nicotina ${ }^{(38)}$.

Por otra parte, en modelos experimentales animales, la exposición crónica a nicotina, causa tolerancia al efecto de la misma sobre la PA, generando un descenso de la misma a través de mediadores de la vasodilatación y la relajación del músculo liso vascular (27). Estos hallazgos, sumados a un menor índice de masa corporal en pacientes fumadores, podrían explicar las cifras de PA más bajas observadas en pacientes tabaquistas en algunos estudios y sustenta lo controversial de esta asociación (38).

\section{J) Diabetes}

La prevalencia de la diabetes en Uruguay, varía en los estudios más recientes entre un $5 \%$ y $14 \%$, hallándose en este estudio una prevalencia más alta del $16 \%{ }^{(39)}$.

\section{K) Asociación Diabetes e HTA Nocturna}

Se constató que el $74.2 \%$ de los pacientes diabéticos tenían HTA nocturna, siendo la misma significativa. Esta asociación puede explicarse en parte por la disfunción autonómica que puede generar la DM ${ }^{(40)}$. La asociación entre HTA y DM ha sido confirmada en otros trabajos. En un estudio multicéntrico que incluyó a 12.765 pacientes, se observó una media de PA sistólica significativamente mayor en los pacientes con DM, encontrándose una prevalencia de HTA nocturna del $90 \%$ en los pacientes hipertensos no controlados con DM ${ }^{(41)}$.

\section{L) IMC}

En Uruguay el $64,8 \%$ de los adultos presentan un IMC aumentado $(37,2 \%$ con sobrepeso y $27,6 \%$ con obesidad), en este estudio se encontraron resultados similares ${ }^{(42)}$. El alto nivel de adiposidad está fuertemente asociado con una elevación de la PA. Por lo tanto, un menor IMC entre los pacientes fumadores, podría ser una de las razones, por la que en algunos estudios se encuentre una asociación inversa entre tabaquismo e HTA ${ }^{(19)}$.

\section{M) Asociación IMC e HTA nocturna}

Se constató un IMC más elevado en los pacientes con HTA nocturna comparados a los que no la presentaban, siendo estas diferencias significativas. EI IMC aumentado se ha asociado previamente al desarrollo de HTA nocturna. Hay estudios que muestran que durante la noche la presión sistólica y diastólica declina mayormente en los pacientes con IMC adecuado comparado a los pacientes obesos. Esta relación entre HTA y obesidad se relaciona a una reabsorción anormal de sodio y agua a nivel renal, alteraciones del filtrado glomerular y activación del sistema simpático en los pacientes obesos ${ }^{(43)}$.

\section{N) SAHOS}

Se encontró una prevalencia menor de SAHOS (8.7\%) que en otros estudios $(24-26 \%$ en hombres y de 17-28\% en mujeres), pudiendo estar asociado al subdiagnóstico de esta patología en la población de estudio y también al tamaño muestral ${ }^{(44)}$.

\section{0) Asociación SAHOS e HTA nocturna}

En este estudio, hubo una mayor prevalencia de HTA nocturna entre los pacientes con SAHOS. Otros trabajos han reportado un incremento agudo de la PA de $20 \mathrm{mmHg}$ relacionados 
a eventos obstructivos, observándose una asociación significativa entre la severidad de SAHOS y HTA. Esto puede estar asociado a una mayor actividad del sistema simpático, activación del sistema renina-angiotensina-aldosterona, estrés oxidativo y mayor componente inflamatorio ${ }^{(45)}$.

Limitaciones del estudio: La recolección de datos sobre factores de riesgo cardiovascular asociados, tales como DM y SAHOS, fue mediante una encuesta, reportada por los participantes del estudio. Esto podría afectar la evaluación de dichas variables, pudiendo existir un subdiagnóstico de estas patologías.

\section{Conclusiones}

En la población en estudio, el grupo de pacientes fumadores se comportó con una tendencia a presentar cifras de presión arterial media diurna sistólica y diastólica más elevadas que los no fumadores, esto sugiere que el tabaquismo podría interferir en el control de cifras de PA.

No se encontró relación entre tabaquismo y patrones de variabilidad de mayor riesgo, como tampoco con HTA nocturna. Sin embargo, se evidenció una asociación entre HTA nocturna con DM e IMC aumentado. El abandono del consumo de tabaco es una de las estrategias fundamentales en el tratamiento de la HTA.

\section{Conflicto de interés}

Los autores declaran no tener conflicto de interés.

No se recibió financiación por el trabajo.

\section{Bibliografía}

1- Fernández de Bobadilla J, Sanz de Burgoa V, Garrido Morales P, López de Sá E. Riesgo cardiovascular: evaluación del tabaquismo y revisión en atención primaria del tratamiento y orientación sanitaria. Estudio RETRATOS. Aten. Prim. 2011;43(11):595-603.

2- World Health Organization - WHO. WHO report on the global tobacco epidemic, 2019: offer help to quit tobacco use [Internet]. Geneva: WHO; 2019 [acceso 02/10/2020]. 209 p. Disponible en: https:// www.who.int/tobacco/global_report/en/.

3- Uruguay. Ministerio de Salud. Encuesta mundial de tabaquismo en adultos: Global Adult Tobacco Survey (GATS) Uruguay 2017. Disponible en: https://www.paho.org/uru/index. php?option=com_docman\&view=download\&alias=576-uru-gats-2017-core-factsheet\&category slug=publications\&ltemid=307 [Acceso: 28/09/2020]

4- Ozemek C, Phillips S, Popovic D, Laddu-Patel D, Fancher I, Arena R et al. Nonpharmacologic management of hypertension. Curr Opin Cardiol. 2017;32(4):381-388.

5- Ursoniu S, Mikhailidis DP, Serban MC, Penson P, Toth PP, Ridker PM, et al. Lipid and Blood Pressure Meta-analysis Collaboration (LBPMC) Group. The effect of statins on cardiovascular outcomes by smoking status: A systematic review and meta-analysis of randomized controlled trials. Pharmacol Res. 2017; 122:105-117.

6- Williams B, Mancia G, Spiering W, Agabiti Rosei E, Azizi M, Burnier M, et al. Guía ESC/ESH 2018 sobre el diagnóstico y tratamiento de la hipertensión arterial. Rev Esp Cardiol. 2019;72(2):160.e1-e78

7- Petermann F., Durán E., Labraña A. M., Martínez M. A., Leiva A. M., Garrido-Méndez A.,et al. Factores de riesgo asociados al desarrollo de hipertensión arterial en Chile. Rev Med Chil. 2017; 145(8), 9961004.

8- O’Donnell CJ, Elosua R. Factores de riesgo cardiovascular. Perspectivas derivadas del Framingham Heart Study. Rev Esp Cardiol. 2008;61(3):299-310.

9- Unger T, Borghi C, Charchar F, Khan NA, Poulter NR, Prabhakaran D, et al. 2020 International Society of Hypertension Global Hypertension Practice Guidelines. J Hypertens. 2020 Jun;38(6):982-1004

10- Ahmad M, Makati D, Akbar S. Review of and Updates on Hypertension in Obstructive Sleep Apnea. Int J Hypertens. 2017;2017:1-13.

11- Smolensky MH, Hermida RC, Ayala DE, Mojón A, Fernández JR. Bedtime Chronotherapy with Conventional Hypertension Medications to Target Increased Asleep Blood Pressure Results in Markedly Better Chrono prevention of Cardiovascular and Other Risks than Customary On-awakening Therapy. Heart Fail Clin. 2017 oct;13(4):775-92. 
12- Sauza-Sosa JC, Cuéllar-Álvarez J, Villegas-Herrera KM, Sierra-Galán LM. Aspectos clínicos actuales del monitoreo ambulatorio de presión arterial [Current clinical aspects of ambulatory blood pressure monitoring]. Arch Cardiol Mex. 2016 Jul-Sep;86(3):255-9. Spanish.

13- Juhanoja EP, Niiranen TJ, Johansson JK, Puukka PJ, Thijs L, Asayama K, et al. Outcome-Driven Thresholds for Increased Home Blood Pressure Variability. Hypertens. 2017 abr;69(4):599-607.

14- Chobanian AV, Bakris GL, Black HR, Cushman WC, Green LA, Izzo JL Jr, et al. The seventh report of the Joint National Committee on Prevention, Detection, Evaluation, and Treatment of High Blood Pressure: the JNC 7 Report. JAMA 2003;289:2560-2572

15- Kim S, Lee J. The association of smoking and hypertension according to cotinine-verified smoking status in 25,150 Korean adults. Clinical and Experimental Hypertension. 2018;41(5):401-408.

16- Gupta R, Gupta S, Sharma S, Sinha D, Mehrotra R. A systematic review on association between smokeless tobacco \& cardiovascular diseases. Indian J Med Res. 2018;148(1):77. http://dx.doi. org/10.4103/ijmr.IJMR_2020_17

17- St George IM, Willians S, Staton WR, Silva PA. Smoking and blood pressure in 15 year olds in Dunedin, New Zealand. Br Med J. 1991; 302: 89-90

18- Karven M, Orma E, Keyes A, et al. Cigarette smoking serum cholesterol, blood pressure and body fatness. Observations in Finland. Lancet 1959; 1: 492.

19- Lefort, M., Neufcourt, L., Pannier, B., Vaïsse, B., Bayat, S., Grimaud, O. and Girerd, X., 2018. Sex differences in adherence to antihypertensive treatment in patients aged above 55: The French League Against Hypertension Survey (FLAHS). J Clin Hypertens, 20(10), pp.1496-1503.

20- Di Giosia P, Giorgini P, Stamerra C, Petrarca M, Ferri C, Sahebkar A. Gender Differences in Epidemiology, Pathophysiology, and Treatment of Hypertension. Curr Atheroscler Rep. 2018; 20(3):13. doi: 10.1007/s11883-018-0716-z.

21- Triunfo P, Harris J, Balsa A. Evaluación de la campaña antitabaco en Uruguay: balance de diez años y desafíos. Rev Panam Salud Pub. 2016 Oct; 40(4):256-262.

22- Rubinstein A., Irazola V., Calandrelli M., Chen C., Gutierrez L., Lanas F., Manfredi J., Mores N., Poggio R., Ponzo J., Seron P., Bazzano L. and He J., 2016. Prevalence, Awareness, Treatment, and Control of Hypertension in the Southern Cone of Latin America. Am J Hypertens. 2016 Dec 1;29(12):1343-1352. doi: 10.1093/ajh/hpw092

23- Hermida R, Crespo J, Domínguez-Sardiña M, Otero A, Moyá A, Ríos M et al. Bedtime hypertension treatment improves cardiovascular risk reduction: the Hygia Chronotherapy Trial. Eur Heart J. 2019; ehz754. doi:10.1093/eurheartj/ehz754

24- Kristanto A, Adiwinata R, Suminto S, Kurniawan BN, Christianty F, Sinto R. Nocturnal hypertension: neglected issue in comprehensive hypertension management. Acta Med Indones. 2016. 48: 76-82.

25- Vyssoulis GP, Marinakis AG, Karpanou EA, Triantaphylou AG, Barbetseas ID, Rizos JK, et al. Smoking and sympathetic system mismatch in arterial hypertension. Am J Hypertens. 2012. 15(S3), 218A.

26- Hermida RC, Ayala DE, Calvo C, Covelo M, Rodriguez M, Fontao MJ, et al. Influence of cigarette smoking on ambulatory blood pressure in patients with essential hypertension. American Journal of Hypertension 2005;18(5 Suppl 1): A33. https://doi.org/10.1016/j.amjhyper.2005.03.087

27- Ebina T. Smoking and Incident Hypertension $\otimes$ Importance of Cotinine-Verified Smoking Status. Circ. J. 2018;82(6):1510-1512.

28- Salazar M, Espeche W, Balbín E, Leiva Sisnieguez C, Minetto J, Leiva Sisnieguez B et al. Prevalence of isolated nocturnal hypertension according to 2018 European Society of Cardiology and European Society of Hypertension office blood pressure categories. J Hypertens. 2020;38(3):434-440.

29- Cho M. Clinical Significance and Therapeutic Implication of Nocturnal Hypertension: Relationship between Nighttime Blood Pressure and Quality of Sleep. Korean Circ J. 2019;49(9):818-828.

30- Musameh M, Nelson C, Gracey J, Tobin M, Tomaszewski M, Samani N. Determinants of day-night difference in blood pressure, a comparison with determinants of daytime and night-time blood pressure. J Hum Hypertens. 2017;31(1):43-48.

31- Mikkelsen KL, Wiinberg N, Høegholm A, Christensen HR, Bang LE, Nielsen PE, et al. Smoking related to 24-h ambulatory blood pressure and heart rate: a study in 352 normotensive Danish subjects. Am J Hypertens. 1997;10(5 Pt 1):483-91 
32- Verdecchia P, Schillacl G, Borgioni C, Ciucci A, Zampi I, Battistelli M et al. Cigarette smoking, ambulatory blood pressure and cardiac hypertrophy in essential hypertension. J Hypertens. 1995;13(10):1209-1216.

33- Sakhuja S, Booth J, Lloyd-Jones D, Lewis C, Thomas S, Schwartz J et al. Health Behaviors, Nocturnal Hypertension, and Non-dipping Blood Pressure: The Coronary Artery Risk Development in Young Adults and Jackson Heart Study. Am. J. Hypertens. 2019;32(8):759-768.

34- Halperin R, Michael Gaziano J, Sesso H. Smoking and the Risk of Incident Hypertension in Middleaged and Older Men. American Journal of Hypertension. 2008;21(2):148-152.

35- Kim S, Lee J. The association of smoking and hypertension according to cotinine-verified smoking status in 25,150 Korean adults. Clinical and Experimental Hypertension. 2018;41(5):401-408.

36- Morillo M, Amato M, Cendon Filha S. Registro de 24 horas da pressão arterial em tabagistas e nãotabagistas. Arq. Bras. Cardiol. 2006;87(4):504-511.

37- Kawano Y. Diurnal blood pressure variation and related behavioral factors. Hypertens Res. 2011;34:281-285

38- Li G, Wang H, Wang K, Wang W, Dong F, Qian Y, et al. The association between smoking and blood pressure in men: a cross-sectional study. BMC Public Health. 2017;17(1):797.

39- Sandoya, E. Diabetes y enfermedad cardiovascular en Uruguay. Rev. Uru. Cardiol. 2016; 31(3), 505514.

40- Rossen N, Knudsen S, Fleischer J, Hvas A, Ebbehøj E, Poulsen P et al. Targeting Nocturnal Hypertension in Type 2 Diabetes Mellitus. Hypertension. 2014;64(5):1080-7.

41- Ayala D, Moyá A, Crespo J, Castiñeira C, Domínguez-Sardiña M, Gomara S et al. Circadian Pattern of Ambulatory Blood Pressure in Hypertensive Patients With and Without Type 2 Diabetes. Chronobiol. Int. 2012;30(1-2):99-115

42- Uruguay. Ministerio de Salud. Dirección General de la Salud. Área Programática Nutrición. Guía Alimentaria para la población uruguaya: para una alimentación saludable, compartida y placentera[Internet]. Montevideo: MS, 2016. [acceso: 28 setiembre 2020] Disponible en: https://www. gub.uy/ministerio-desarrollo-social/comunicacion/publicaciones/guia-alimentaria-para-la-poblacionuruguaya

43- Kotsis V, Stabouli S, Bouldin M, Low A, Toumanidis S, Zakopoulos N. Impact of Obesity on 24-Hour Ambulatory Blood Pressure and Hypertension. Hypertension. 2005;45(4):602-7.

44- Torres G, Sánchez-de-la-Torre M, Barbé F. Relationship Between OSA and Hypertension. Chest. 2015;148(3):824-832.

45- Phillips CL, O’Driscoll DM. Hypertension and obstructive sleep apnea. Nat Sci Sleep. 2013; 5:43-52.

\section{Aporte cada autor al trabajo}

Carolina Acosta: concepción y diseño del trabajo, recolección de datos, análisis estadístico de los datos, análisis e interpretación de los resultados, redacción del manuscrito.

Paola Spósito: concepción y diseño del trabajo, análisis estadístico de los datos, análisis e interpretación de los resultados, redacción del manuscrito, revisión crítica del manuscrito.

Verónica Torres Esteche: concepción y diseño del trabajo, análisis estadístico de los datos, análisis e interpretación de los resultados, redacción del manuscrito, revisión crítica del manuscrito.

Florencia Sacchi: concepción y diseño del trabajo, recolección de datos, análisis e interpretación de los datos.

Lucía Pomies: concepción y diseño del trabajo, recolección de datos, análisis e interpretación de los datos.

Maximiliano Pereda: recolección de datos, análisis e interpretación de los datos.

Sergio Viña: concepción y diseño del trabajo, análisis e interpretación de los resultados.

Enrique Soto: concepción y diseño del trabajo, análisis e interpretación de los resultados. 